\title{
THREE THEOREMS ON NORMAL ORTHOGONAL SETS
}

BY M. H. STONE

The three theorems which we desire to record here may be of some interest, in spite of their simplicity. So far as the author knows, they have not appeared in the literature. On the other hand, the corollaries appended are to be found in the journals cited in the footnotes, but were there obtained in different fashion. Throughout our work we shall assume on the part of the reader some familiarity with the theorem of Riesz-Fischer and with convergence in the mean.* All functions considered are understood to be real functions defined on the interval $(a, b)$. They are to be summable with summable square in the Lebesgue sense on this interval. The first result is embodied in the following theorem.

Theorem I. A necessary and sufficient condition that every pair of functions $f_{1}, f_{2}$ having identical Fourier coefficients with respect to a normal orthogonal set $\left\{u_{k}\right\}$ should have identical coefficients with respect to a second set $\left\{v_{k}\right\}$ is that the formal series for each function of the set $\left\{v_{k}\right\}$ in terms of $\left\{u_{k}\right\}$ should converge in the mean to that function.

The condition is necessary. We write

$$
\int_{a}^{b} v_{i} u_{k}=c_{i k}
$$

so that the formal series for $v_{i}$ is $\sum_{k=1}^{\infty} c_{i k} u_{k}$. Suppose that for some fixed $i$ the series does not converge in the mean

* Plancherel, Rendicontr di Palermo, vol. 30 (1910), pp. 289-335, especially pp. 290-297. See also Riesz, Göttrnater Nachrichten, 1907, p. 117. 
to $v_{i}$; that is, that

$$
\sum_{k=1}^{\infty} c_{i k}^{2}<\int_{a}^{b} v_{i}^{2}
$$

By the Riesz-Fischer theorem, the series converges in the mean to a second function $v_{i}^{*}$ for which we have

for all $k$. Now

$$
\int_{a}^{\bullet b} v_{i}^{*} u_{k}=c_{i k}
$$

$$
\lim _{K \rightarrow \infty} \int\left(v_{i}^{*}-\sum_{k=1}^{K} c_{i k} u_{k}\right) v_{i}=0
$$

as we see by the use of Schwarz's inequality. In other words

$$
\int_{a}^{b} v_{i}^{*} v_{i}=\sum_{k=1}^{\infty} c_{i k}^{2} \neq \int_{a}^{b} v_{i} v_{i} .
$$

The functions $v_{i}$ and $v_{i}^{*}$ have identical coefficients with respect to $\left\{u_{k}\right\}$ but different coefficients with respect to the set $\left\{v_{k}\right\}$. This contradicts the hypothesis, and therefore shows the necessity of the condition.

The condition is sufficient. We write $\int_{a}^{b} f_{1} u_{k}=\int_{a}^{b} f_{2} u_{k}=a_{k}$. We use the Schwarz inequality to prove that

$$
\begin{aligned}
& \lim _{K \rightarrow \infty} \int_{a}^{b b} f_{1}\left(v_{i}-\sum_{k=1}^{K} c_{i k} u_{k}\right)=0, \\
& \lim _{K \rightarrow \infty} \int_{a}^{b} f_{2}\left(v_{i}-\sum_{k=1}^{K} c_{i k} u_{k}\right)=0 .
\end{aligned}
$$

It follows that $\int_{a}^{b} f_{1} v_{i}=\int_{a}^{b} f_{2} v_{i}=\sum_{k=1}^{\infty} c_{i k} a_{k}$. This completes the proof of Theorem I.

We shall next prove the following theorem.

Theorem II. A necessary and sufficient condition that the formal Fourier series $\sum_{k=1}^{\infty} a_{k} u_{k}, \sum_{k=1}^{\infty} b_{k} v_{k}$ associated with an arbitrary function $f$ should converge in the mean to the same function $f^{*}$ is that the formal series for each function of the set $\left\{v_{k}\right\}$ in terms of the set $\left\{u_{k}\right\}$ should converge in the mean to that function and vice versa. 
The condition is necessary. This fact appears as obvious as soon as we set $f=u_{k}, f=v_{k},(k=1,2, \cdots, \infty)$.

The condition is sufficient. The series $\sum_{k=1}^{\infty} a_{k} u_{k}$ converges in the mean to a function $f_{1}^{*}$, the series $\sum_{k=1}^{\infty} b_{k} v_{k}$ to a function $f_{2}^{*}$, by the theorem of Riesz-Fischer. We now appeal to Theorem I to show that

$$
\int_{a}^{b} f u_{k}=\int_{a}^{b} f_{1}^{*} u_{k}=\int_{a}^{b} f_{2}^{*} u_{k},(k=1,2, \cdots, \infty) .
$$

Since $f, f_{2}^{*}$ have identical coefficients with respect to $\left\{v_{k}\right\}$, they also have identical coefficients with respect to $\left\{u_{k}\right\}$ by Theorem I. The equalities above then result. In the same manner we show that

$$
\int_{a}^{b} f v_{k}=\int_{a}^{b} f_{1}^{*} v_{k}=\int_{a}^{b} f_{2}^{*} v_{k}, \quad(k=1,2, \cdots, \infty) .
$$

Next, from Bessel's inequality, we obtain

$$
\sum_{k=1}^{\infty} a_{k}^{2}=\int_{a}^{b} f_{1}^{* 2} \geq \sum_{k=1}^{\infty} b_{k}^{2}, \sum_{k=1}^{\infty} b_{k}^{2}=\int_{a}^{b} f_{2}^{* 2} \geqq \sum_{k=1}^{\infty} a_{k}^{2} .
$$

The inequalities are therefore impossible. We see that either of the two series $\sum_{k=1}^{\infty} a_{k} u_{k}, \sum_{k=1}^{\infty} b_{k} v_{k}$ converges in the mean to each of the two functions $f_{1}^{*}, f_{2}^{*}$. The two functions are therefore the same, in the sense that they can differ at most on a set of zero measure. This completes the proof of Theorem II.

The two corollaries which we shall now discuss are to be found in the literature.

Coroldary I.* A necessary and sufficient condition that a normal orthogonal set $\left\{u_{k}\right\}$ be closed is that the formal series for each function of an arbitrary closed normal orthogonal set $\left\{v_{k}\right\}$ in terms of $\left\{u_{k}\right\}$ converge in the mean to that function.

In order that $\left\{u_{k}\right\}$ be a closed set, it is necessary and sufficient that every function represented by the convergence

* Lauricella, Rendicontr dei Lincei, (5), vol. 21 (1912), pp. 675-85. 
in the mean of its formal series in terms of the closed set $\left\{v_{k}\right\}$ be so represented in terms of $\left\{u_{k}\right\}$. Theorem II then gives us the last link in the proof.

Corollary II.* Let $\left\{\boldsymbol{\Phi}_{k}\right\}$ be a set of functions such that the system of equations $\int_{a}^{b} \theta \Phi_{k}=0, k=1,2, \cdots, \infty$, implies that the function $\theta$ is identically zero except possibly on a set of zero measure. Then a necessary and sufficient condition that a normal orthogonal set $\left\{u_{k}\right\}$ be closed is that the formal series for each function of the set $\left\{\boldsymbol{\Phi}_{k}\right\}$ in terms of $\left\{u_{k}\right\}$ converge in the mean to that function.

By Schmidt's process of normalization and orthogonalization we form from the set $\left\{\Phi_{k}\right\}$ a closed normal orthogonal set $\left\{v_{k}\right\}$. Each function of $\left\{v_{k}\right\}$ is a linear combination with constant coefficients of a finite set of functions of $\left\{\boldsymbol{\Phi}_{k}\right\}$, and conversely. We recall that series which can be summed in the sense that they converge in the mean can be added term by term. By using Corollary I in conjunction with this fact we complete our demonstration.

Of particular interest under the second corollary is the special case obtained by setting $\boldsymbol{\Phi}_{k}=x^{k-1}, k=1,2, \cdots, \infty$. For further remarks on these two corollaries we refer to the papers already mentioned.

A theorem very similar to Theorem II follows.

Theorem III. A necessary and sufficient condition that the difference of the two formal series $\sum_{k=1}^{\infty} a_{k} u_{k}, \sum_{k=1}^{\infty} b_{k} v_{k}$ associated with an arbitrary function $f$ should converge in the mean to zero, is that the formal series for each function of $\left\{v_{k}\right\}$ in terms of $\left\{u_{k}\right\}$ converge in the mean to that function, and vice versa.

The condition is necessary. We can see this fact readily by putting $f=u_{k}, f=v_{k},(k=1,2, \cdots, \infty)$.

The condition is sufficient. We know by Theorem II that the two series converge in the mean to the same

* Severini, Rendiconti di Palermo, vol. 36 (1913), pp. 177-202, especially pp. 194-95. 
function $f^{*}$. Hence their difference converges in the mean to zero.

This last theorem may be looked upon as giving a necessary and sufficient condition that two normal orthogonal sets $\left\{u_{k}\right\},\left\{v_{k}\right\}$ be equivalent in the sense that the formal series for an arbitrary function in terms of them always converge in the mean to the same function.

New York City

\section{ON THE COMPLE'TE INDEPENDENCE OF THE FUNCTIONAL EQUATIONS OF INVOLUTION*}

BY C. C. MAC DUFFEE

1. Introduction. The three fundamental theorems or "laws" of involution are commonly written

I $a^{m} \cdot a^{n}=a^{m+n}, \quad$ II $\left(a^{m}\right)^{n}=a^{m n}$, III $a^{n} \cdot b^{n}=(a b)^{n}$.

In $\S 2$ of this paper these equations are abstractly formulated as functional equations. In $\S 3$ it is proved that any function satisfying Equations I and II also satisfies III, provided certain underlying conditions $A$ and $B$ are fulfilled.

In $\S 4$ a number system $\mathfrak{R}$ is introduced whose elements are the numbers $[\xi, r]$ where $\xi$ and $r$ are real numbers. Two operations, addition and multiplication, are introduced, and it is shown that these operations obey all the ordinary laws of algebra except the associative law of addition. This number system $\mathfrak{N}$ is then used in the discussion of the complete independencet of Equations I, II and III.

* Presented to the Society, April 28, 1923.

$\dagger$ Consider $n$ conditions. There are conceptually $2^{n}$ distinct cases to be considered according as Condition 1 holds or does not hold, Condition 2 holds or does not hold, ..., Condition $n$ holds or does not hold. If none of these $2^{n}$ cases is empty, the $n$ conditions are said to be completely independent. Cf. E. H. Moore, The New Haven Mathematical Colioquium, (Yale University Press, 1910), pp. 81, 82 